\title{
Effect of Trunk Extensor Muscle Fatigue on Postural Stability in Women Undergone Lower Segment Cesarean Section
}

\author{
Rutuja Malavde', Amrutkuvar Rayjade \\ ${ }^{1}$ Intern, Krishna College Of Physiotherapy, Krishna Institute Of Medical Sciences, Karad, ${ }^{2}$ Associate Professor, \\ Department Of Musculoskeletal Sciences, Krishna College Of Physiotherapy, Krishna Institute Of Medical \\ Sciences, Karad
}

\begin{abstract}
Background: Cesarean delivery involves thorough disruption of the anterior abdominal wall and is related with greater reports of postpartum pain and slower more painful recovery than vaginal birth. An increase in the inter-recti distance puts postural stability in hazard. It also weakens the abdominal muscles and influence their functions. This can result in an altered trunk mechanics, impaired pelvic stability and postural change, that leaves the lumbar spine and pelvis more susceptible to injury. Low endurance of back and hip muscles has been reported in women after delivery which can result into pelvic girdle pain and lumbar pain. There is lack of research on this topic, indeed this made us to study the effect of trunk extensor muscle fatigue on postural stability in women undergone lower segment cesarean section.
\end{abstract}

Objectives: To find the effect of trunk extensor muscle fatigue on postural stability in women undergone lower segment cesarean section.

Method: In this observational study, 37 women undergone cesarean section in age group 22 to 35 years were included. One- Leg Standing Balance Test and Functional Reach Test were the major outcome measures to assess postural stability in three conditions: No fatigue, Fatigue and Recovery Condition. Borg 6-20 scale was used to check the exertion level after inducing fatigue in women. Later, data was collected and analysis was done.

Result: Both One- Leg Standing Balance Test and Functional Reach Test showed a decrease from No fatigue condition to Fatigue condition. The results indicate that postural stability was significantly reduced in the Fatigue condition as compared to the No fatigue condition.

Conclusion: Trunk extensor muscle fatigue significantly reduces the postural stability in women undergone lower segment cesarean section.

Keywords: Cesarean Section, Postural stability, Muscle fatigue, One-leg standing balance test, Functional reach test.

\section{Corresponding Author:}

\section{Dr. Amrutkuvar Rayjade}

Associate Professor, Department Of Musculoskeletal Sciences, Krishna College Of Physiotherapy, Krishna Institute Of Medical Sciences, Karad, Maharashtra, India, 415110

Contact Number: 8169395616

e-mail: mr250615@gmail.com

\section{Introduction}

Postural control is a complicated task which requires the integration of visual, vestibular and somatosensory inputs from all over the body to determine the position and motion of the body in pace and also the ability to generate forces to control body position ${ }^{1}$.

Muscle fatigue is an acute and activity- induced decline in the muscle force or muscle power ${ }^{2}$. Muscle 
fatigue is an important condition which comprises of sensorimotor integration and hence, postural control. Postural muscle fatigue such as trunk extensors may occur in many labour conditions demanding high tension in the muscle group ${ }^{3}$. Such tension represents an important accommodating factor of the spinal alignment and stability produced lumbar discomfort or pain ${ }^{4}$.

As there is an occurrence of changes in a pregnant women, static stability may get affected due to gestational weight gain and its assymetrical distribution in the anterior abdominal region ${ }^{5}$, adaptive postural changes necessary for the antero posterior center of gravity (COG) location readjustment ${ }^{6}$, as well as the increased joint laxity ${ }^{7}$, also there is a change in the transient stability in the postpartum period due to increased connective tissue laxity and altered posture ${ }^{8}$. Along with the pregnant women, postpartum women's postural stability may also get affected due to inadequate sleep and sleep deprivation ${ }^{9}$. There are many other factors that may affect the abdominal muscle function after pregnancy, including the mode of delivery and physical activity levels. Cesarean delivery involves thorough disruption of the anterior abdominal wall and is related with greater reports of postpartum pain and slowers more painful recovery than vaginal birth ${ }^{10}$.

There are several physiological processes occurring during pregnancy that has an impact on the mother's musculoskeletal system. There is a substantial stretch of the abdominal muscles and common occurrence of increase in the inter-recti distance ${ }^{11}$

Throughout the mother's body hormones act on connective tissue, which result in joint laxity, particularly in pelvis ${ }^{12}$. There is an increase in the importance and role of muscular stabilization, which is provided by the core muscles including, abdominal muscles ${ }^{13}$.

After pregnancy many women experience an increase in the inter-recti abdominal muscle distance as a result of stretching and thinning of the linea alba ${ }^{14}$. A widening of $>2.7 \mathrm{~cm}$ at the level of umbilicus is considered as a pathological diastasis of therectus abdominis muscle (DRAM $)^{15}$. Hormonal elastic changes of the connective tissue, mechanical stresses placed on the abdominal wall by the growing fetus and the displacement of abdominal organs can lead to occurrence of DRAM ${ }^{16}$. It is very common and it can have negative health consequences for women after pregnancy. The abdominal wall plays an important role in maintaining posture, trunk and pelvic stability, respiration, trunk movement and abdominal viscera support. An increase in the inter- recti distance puts this function in hazard ${ }^{17}$. It also weakens the abdominal muscles and influence their functions ${ }^{18}$. This can result in an altered trunk mechanics, impaired pelvic stability and postural change, that leaves the lumbar spine and pelvis more susceptible to injury ${ }^{19}$.

DRAM is quiet common and is associated with the risk factors such as multiparity, maternal age and childcare responsibilities. There is an opposing evidence linking DRAM with weight gain and high body mass index $(\mathrm{BMI})^{20}$.

Laxity in the supporting tissue is the source of muscle strain during actual birth. During Cesarean delivery, the lower back muscles are used, along with pelvic muscles and abdominals ${ }^{21}$. Low endurance of back and hip muscles has been reported in women after delivery which can result into pelvic girdle pain and lumbar pain ${ }^{22}$. Consequently, this study focuses on how trunk extensor muscle fatigue affects postural stability in postpartum period.

\section{Material and Methodology}

This study was done to find the effect of trunk extensor muscle fatigue on postural stability in women undergone lower segment cesarean section. The study was carried out in Krishna Physiotherapy Out Patient Department, Karad. An approval of the study was obtained from the institutional ethical committee of Krishna Institute Of Medical Sciences Deemed University. The purpose of the study was explained to the participants and consent form was taken. 37 women who underwent lower segment cesarean section was taken from Karad forstudy. The inclusioncriterias were women undergone single Cesarean section 6 months back in age group between 22 to 35 years and those who do not perform core muscle exercise on regular basis. The exclusioncriterias were those women who underwent multiple cesarean section, having any musculoskeletal dysfunction and those having any psychological problem. One- leg Standing Balance Test and Functional Reach Test were the major outcome measures to assess postural stability. Order of testing was One- leg standing balance test followed by Functional reach test under three experimental conditions, No fatigue, Fatigue and Recovery. The dominant leg was first determined by asking the subject to kick a ball placed on the floor in front of her and the 'kicking limb' was considered 
as 'dominant leg'.For One- leg standing balance test, subject was standing barefoot on a firm stable surface on the dominant leg with the other leg raised, arms crossed over the chest. Test was performed under eyes closed condition to avoid vision from interfering with the task of postural control. Time of one- leg standing was recorded in seconds using a stop watch. Test was terminated when the women had used her arms (i.e. uncrossed arms), used the raised foot (moved it towards or away from the standing limb or touched the floor), moved the weight bearing foot to maintain balance (i.e. rotated foot on the ground), or opened eyes. Three trials were given and best of them were taken as the final reading. Functional reach test was performed by the subject standing next to but not touching a wall with dominant arm closer to the wall at 90 degrees of shoulder flexion with a closed fist. Women had selected a comfortable stance with feet shoulder width apart. To keep foot positions and base of support constant, during reassessments in all three conditions, footprints were marked. The subject was asked to reach forward as far as possible without taking a step. The distance between the start and the end position was measured using the third metacarpal as the reference point and was recorded in centimeters. Three trials were given and the average of the three trials was taken as the final reading. To induce fatigue of trunk extensor muscles subject was made to perform dynamic trunk extensions until maximum exhaustion. For this task subject lied prone on a bench with the upper body unsupported in the horizontal plane and the lower limb secured to the bench with straps at the hips, knees and ankles. During the test, arms were held across the chest. The subject was instructed to raise her upper body to a horizontal position with the head and neck in neutral position and then lowering it back down. Time of $40 \mathrm{beeps} / \mathrm{min}$ was recorded using digital metronome application to ensure that the subjects perform the extensions at a consistent rate. Women were instructed to perform the extension movement as many times as possible till she was unable to continue due to muscle fatigue. After termination of the task postural stability was assessed immediately (Fatigue condition) using One- leg standing balance test and Functional reach test as described earlier. The subjective exertion level for the fatiguing task was assessed through the Borg 6-20 scale. For recovery of induced trunk extensor muscle fatigue, rest was given in a supported position. Further, recovery time was noted and to observe recovery in postural stability (Recovery condition) One- leg standing balance test and Function reach test was performed again and readings were noted. Women were asked to refrain from any strenuous physical activity for past 48 hours. Then, statistical analysis and interpretation was done for each candidate to find out the effect of trunk extensor muscle fatigue on postural stability in women undergone lower segment cesarean section.

\section{Statistical Analysis:}

For sampling size following formula was used

$$
\begin{aligned}
& \mathbf{n}=\mathbf{4 p q} / \mathbf{l}^{\mathbf{2}} \\
& (p=70 \%, q=30 \% \text { and } L=15 \%) \\
& \mathbf{4} \times \mathbf{7 0} \times \mathbf{3 0} / \mathbf{2 2 5}=\mathbf{3 7} \\
& \mathbf{n}=\mathbf{3 7}
\end{aligned}
$$

Statistical analysis of the recorded data was done by using the software INSTAT App. Unpaired t test was used to determine $p$ value $(<0.0001)$, which indicates it is extremely significant. Tukey Kramer Multiple Comparison Test was used to compare postural stability in three conditions: No fatigue, Fatigue and Recovery condition.

\section{Result}

Table No 1: Test Results

\begin{tabular}{|l|c|c|c|c|}
\hline Tests & Mean & Standard Deviation & p Value & Interference \\
\hline Age & 31.43 & 3.087 & $<0.0001$ & Extremely Significant \\
\hline BMI & 23.73 & 4.86 & $<0.0001$ & Extremely significant \\
\hline OLST & 12.736 & 2.954 & $<0.0001$ & Extremely Significant \\
\hline & 8.059 & 2.245 & & \\
\hline & 11.566 & 3.260 & & \\
\hline
\end{tabular}




\begin{tabular}{|l|c|c|c|c|}
\hline Tests & Mean & Standard Deviation & p Value & Interference \\
\hline FRT & 36.257 & 5.523 & $<0.0001$ & Extremely Significant \\
\hline & 27.162 & 5.286 & & \\
\hline & 34.046 & 5.431 & & \\
\hline Borg's Scale & 13.757 & 2.278 & $<0.0001$ & Extremely Significant \\
\hline Recovery Time & 4.659 & 2.121 & $<0.0001$ & Extremely Significant \\
\hline
\end{tabular}

Participants mean age, BMI were 31.43 years and $23.73 \mathrm{~kg} / \mathrm{m}^{2}$ respectively. Mean rating of perceived exertion for the fatiguing task was $13.75+2.278$ on Borg 6-20 scale. Statistical significance was set at $\mathrm{P}<0.0001$. Tukey Kramer Multiple Comparison Test was used to compare postural stability in three conditions: No fatigue, Fatigue and Recovery condition. OLST showed a decrease from No fatigue condition $(12.736+2.954$ seconds) to Fatigue condition $(8.059+2.245$ seconds $)$. FRT showed a decrease from No Fatigue $(36.25+$ 5.52 centimeters $)$ to Fatigue condition $(27.16+5.28$ centimeters). The results indicate that postural stability was significantly reduced in the Fatigue condition as compared to the No fatigue condition.

To determine the recovery period for postural stability, mean value of the consecutive readings for OLST and FRT taken during the recovery condition were compared with the No Fatigue condition. It was observed that mean recovery time for OLST and FRT was $4.65+2.12$ minutes. Further analysis showed no significant difference between the recovery time of oneleg standing test and functional reach test (two- tailed $\mathrm{P}$ $=0.999$ ).

\section{Discussion}

The purpose of the present study was to find out the effect of trunk extensor muscle fatigue on postural stability in women undergone lower segment cesarean section. Trunk extensor muscle fatigue significantly affected postural stability as indicated by reduction in One- leg standing balance test and Functional reach test respectively from No fatigue to Fatigue condition. This result confirms our null hypothesis in accordance with previous reports.

Trunk extensors are mainly postural muscles which are involved in many labour conditions demanding high tension in the muscle group. The erector spinae and multifidus are used to maintain the body posture.
Hence, fatigue in these muscles may cause difficulties in maintaining upright posture. The objective of this study was to find out the effect of trunk extensor muscle fatigue on postural stability in women undergone lower segment cesarean section.

In the present study the mean age group was 31.43 years. Although the age group included in this study was in between 22 to 35 years, out of 37 women postural stability of those of age group in between 30 to 35 years was affected. This may be due to following reasons: small sample size, late marriage, late pregnancy. Late marriage could be due to educational factor, selfdependency, cast discrimination, cultural discrimination, etc.Late pregnancy could be due to various reasons such as sedentary lifestyle, dietary factors, stress, etc. ${ }^{23}$

Out of 37 women, 26 were with normal BMI, 7 were overweight whereas 4 were obese. Participants mean BMI was $23.73 \mathrm{~kg} / \mathrm{m}^{2}$ respectively. This may be due to the hormonal elastic changes of the connective tissue, mechanical stresses placed on the abdominal wall and the displacement of abdominal organs that can lead to occurrence of diastasis rectus abdominis muscle (DRAM). ${ }^{16}$ There is an opposing evidence linking DRAM with weight gain and high body mass index (BMI). ${ }^{20}$

The mean period of LSCS was 7.78 years out of 37 women. This may be due to small sample size, elective cesarean delivery, medical reasons, etc.

Mean recovery time for OLST and FRT was $4.65+2.12$ minutes as in Cesarean delivery there is thorough disruption of the anterior abdominal wall ${ }^{10}$ which causes lack of strength in the abdominal muscles. This results in weakening of the core muscles that ultimately leads to muscle fatigue. Also, it is related with greater reports of postpartum pain and slowers more painful recovery than vaginal birth. ${ }^{10}$ Postural stability may get affected due to gestational weight gain, 
increased connective tissue laxity and altered posture in the postpartum period. Consequently, this study focuses on how trunk extensor muscle fatigue affects postural stability in postpartum period.

\section{Conclusion}

Trunk extensor muscle fatigue significantly reduces the postural stability in women undergone lower segment cesarean section. Postural stability in No Fatigue and Recovery condition was similar as compared to Fatigue condition.

Conflict Of Interest: The authors declare that there are no conflicts of interest concerning the content of the present study.

Source Of Funding: Self- funded.

\section{Ethical Clearance:}

\section{References}

1. Massion J (1992) Movement, posture and equilibrium: interaction and coordination. Prog Neurobiol 38: 35-56.

2. Hunter SK. Performance fatigability: mechanics and task specificity. Cold Spring SHarbPerspect Med.2017. [Epub ahead of print]. doi:10.1101/ cshperspect.a029728.

3. Magnusson, M.; Granqvist, M.; Jonson, R.; Lindell, V.; Lundberg, U.; Wallin, L.; Hansson, T. The loads on the lumbar spine during work at the assembly line. The risks for fatigue injuries of vertebral bodies. Spine (Phila. Pa. 1976) 1990, 15, 774-779. [CrossRef].

4. Freitas, K.P.N.; de Barros, S.S.; Angelo, R.d.C.d.O.; Uchoa, E.P.B.L. Lombalgia occupational e a posturasentada: Efeitos da cenesioterapialaboral. Rev. Dor. 2011, 12, 308-313. [CrossRef].

5. Jensen RK, Doucet S, Treitz T (1996) Changes in segment mass and mass distribution during pregnancy. J Biomech 29: 251-256. PMID: 8849820

6. Whitcome KK, Shapiro LJ, Lieberman DE (2007) Fetal load and the evolution of lumbar lordosis in bipedal hominis. Natre 450: 1075-1078. PMID: 18075592

7. Marnach ML, Ramin KD, Ramsey PS, Song S Stensland JJ, An KN (2003) Characterization of the relationship between joint laxity and maternal hormones in pregnancy. ObstetGynecol 101: 331336.

8. Gilleard WL, Crosbie J, Smith R (2002) Static trunk posture in sitting and standing during pregnancy and early postpartum. Arch Phys Med Rehabil 83: 1739-1744. PMID: 12474180

9. Ma J, Yao YJ, Ma RM, Li JQ, Wang T, Li XJ, et al. (2009) Effects of sleep deprivation on human postural control, subjective fatigue assessment and psychomotor performance. J Int Med Res 37: 1311 1320. PMID: 19930836

10. Lobel M, DeLuca RS. Psychosocial sequelae of cesarean delivery: review and analysis of their causes and implications. Soc Sci Med. 2007;64:2272-84.

11. Boissonnault JS, Blaschak MJ. Incidence o diastasis recti abdominis during the childbearing year. Phys Ther. 1988;68(7):1082-6.

12. Kristiansson P. Svardsudd K, von Schoultz B. Reproductive hormones and aminoteminalpropeptide of type III procollagen in serum as early markers of pelvic pain during late pregnancy. Am J Obstet Gynecol. 1999;180(1):12834.

13. Nemann DA. Kinsiology of the Musculoskeletal System: Foundations fprRehabilitatin. Third ed. St. Louis, MO: Mosby Elsevier, 2016.

14. Hsia $\mathrm{M}$, Jones $\mathrm{S}$. Natural resolution of rectus abdominis diastasis. Two single case studies. Aust J Physiother 2000;46:301-7.

15. Rath AM, Attali P, Dumas JL, Goldlust D, Zhang J, Chevrel JP. The abdominal linea alba: an anatomo-radiologic and biomechanical study. SurgRadiolAnat 1996;18:281-8.

16. Lo T, Candido G, Janssen P. Diastasis of the recti abdominis in pregnancy: risk factors and treatment. Physiother Can 1999;51:32-6,44.

17. Bursch SG. Interrater reliability of diastasis recti abdominis measurement. Phys Ther 1987;67:10779.

18. Fast A. Weiss L. Ducommun EJ, Medina E, Butler JG. Low-back pain in pregnancy. Abdominal muscles, sit-up performance and back pain. Spine (Phila Pa 1976) 1990;15:28-30.

19. Gilleard W, Brown J. Structure and function of the abdominal muscles in primigravid subjects during pregnancy and the immediate postbirth period. 
Phys Ther 1996;76:750-62.

20. Candido G, Lo T, Jansen P. Risk factors for diastasis of the recti abdominis. J Assoc Chart Physiother Womens Health 2005;97:49-54.

21. Hala M. Hanfy et al, Evaluatio of Lumbo-pelvic Stabilising Exercises in the Treatment of Backache after Normal Labor, Journal of American Science, 7 (2), 2011,270-276.
22. Annelie Gutke, Hans Christian Ostgaard and Birgitta Oberg, Association between muscle function and low back pain in relation to pregnancy. J Rehabil Med, 40(4),2008, 304-311.

23. Kumar R, Robson K. A prospective study of emotional disorders in childbearing women. $\mathrm{Br} \mathrm{J}$ Psychiatry 1984; 144: 35-47. 